\title{
Comparison of outcomes of primary and secondary implantation of scleral fixated posterior chamber intraocular lens
}

\author{
V Y W Lee, H K L Yuen, A K H Kwok
}

Br J Ophthalmol 2003;87:1459-1462

\begin{abstract}
Aim To assess and compare the results of primary and secondary implantation of scleral fixated posterior chamber intraocular lens (SFIOL).

Methods: The medical records of a consecutive series of 55 eyes of 55 patients with SFIOLs implanted during (group 1) or after (group 2) complicated senile cataract surgery were retrospectively reviewed and analysed.

Results: There were 30 and 25 eyes in group 1 and 2, respectively. Follow up was from 6 to 36 months. Mean logMAR postoperative best corrected visual acuity in group 1 was not significantly different $(0.50$ (SD 0.36)) from that of group $2(0.36(0.21))(p=0.109)$. Postoperative best corrected visual acuity of $6 / 12$ or better was achieved in $58.6 \%$ and $76.0 \%$ in group 1 and 2 , respectively. The difference was not statistically significant $(p=0.177)$. In group 1, $25(83.3 \%)$ eyes had a total of 55 early complications, while in group 2, 16 (64\%) eyes had 26 early complications $(p=0.028)$. The difference in early complication was statistically significant. For late complication after 1 month, 21 (70.0\%) eyes had a total of 37 complications in group 1, while 13 eyes $(52.0 \%)$ had 19 complications in group $2(p=0.077)$. The difference in late complication was not statistically significant.

Conclusion: Secondary implantation of SFIOL after cataract extraction seems to have a lower early complication rate than primary implantation in complicated cataract extraction although the final visual acuity and late complication rate are not significantly different.
\end{abstract}

I $\mathrm{n}$ the presence of a posterior capsule tear during cataract extraction, the intraocular lens (IOL) can be placed in the sulcus if the capsular rim is available or in the bag if the tear is small. When the posterior capsule tear is large or the capsular rim is unstable, scleral fixated intraocular lenses (SFIOL) or anterior chamber intraocular lenses (ACIOL) can be implanted. No consensus currently exists on the optimal method for IOL implantation without capsular support. Some surgeons prefer to implant SFIOL in the absence of posterior capsular support while others prefer ACIOL. ${ }^{2}$ There are certain advantages of SFIOL over ACIOL such as less corneal endothelial damage, minimising aniseikonia in contralateral eyes that are phakic or pseudophakic with a posterior chamber IOL in place. ${ }^{2-5}$ We use SFIOL when the posterior capsule or its remnants do not allow posterior chamber implantation of IOL. However, the optimal timing for the implantation of SFIOL is not well defined.

In this study we assessed and compared the outcomes of primary and secondary implantation of SFIOL in eyes with complicated senile cataract surgery.

\section{METHODS}

The medical records of all eyes receiving SFIOL with complicated cataract surgery from September 1997 to March 2002 in Hong Kong Eye Hospital, Hong Kong, were retrospectively studied. Institutional ethical approval was not required. Those eyes with dislocated cataract that required pars plana vitrectomy, preoperative diagnosis other than senile cataract, follow up period of less than 6 months, or inadequate medical record were excluded. Patient demographics, preoperative and postoperative Snellen best corrected visual acuity (BCVA), length of follow up, nature and complication of the cataract surgery, nature and any complication of the SFIOL surgery, as well as any postoperative complication were particularly noted.

The Snellen BCVA was converted into logarithm of the minimum angle of resolution (logMAR) units for statistical analysis. ${ }^{6}$ Visual acuity of hand movements and light perception was arbitrarily assigned equivalent of 1.7 and 1.8 $\log$ MAR units, respectively.

For statistical analyses, $\chi^{2}$ test and sample $t$ tests were performed. A p value of 0.05 or less was considered to be statistically significant.

\section{RESULTS}

Fifty five eyes of 55 patients with SFIOLs implanted during (group 1) or after (group 2) complicated senile cataract surgery were included in the analysis. There were 30 and 25 eyes in group 1 and 2, respectively. The choice of primary or secondary SFIOL depended on the patient's preference, corneal clarity, length of operation, and the preference of individual surgeon.

Patient demographics were tabulated in Table 1. Patient age at cataract surgery was $76.2(7.3)$ years (mean (SD)) with a range of 62-92 years in group 1. Mean patient age at secondary SFIOL surgery was 75.5 (7.1) years with a range of 61-87 years in group 2. Mean follow up period was 17.5 (8.2) months with a range of 6-36 months in group 1 . Mean follow up period was 20.9 (8.7) months after secondary SFIOL implantation with a range of 9-36 months in group 2 .

In group 1 , an SFIOL was implanted primarily during cataract surgery because of posterior capsule complications encountered during extracapsular cataract extraction (ECCE) in 12 eyes $(40.0 \%)$ and phacoemulsification in 18 eyes $(60.0 \%)$, respectively. Capsular complications included 23 $(76.7 \%)$ posterior capsule ruptures and vitreous loss, five (16.7\%) zonular dialyses, and two $(6.7 \%)$ loss of whole capsule as a result of unplanned intracapsular cataract extraction (ICCE). In group 2, all 25 aphakic eyes had previous complicated cataract extraction that included ECCE in $17(68.0 \%)$ eyes and phacoemulsification in eight $(32.0 \%)$ eyes. Capsular complications in this group included 20 $(80.0 \%)$ posterior capsule ruptures and vitreous loss, four (16.0\%) zonular dialyses and one $(4.0 \%)$ loss of whole 
Table 1 Patient demographics $(n=55)$

\begin{tabular}{llll}
\hline & Group $\mathbf{1}(\mathbf{n}=\mathbf{3 0})$ & Group $\mathbf{2}(\mathbf{n}=\mathbf{2 5})$ & Statistical test \\
\hline Mean age (SD) (years) & $76.2(7.3)(62-92)$ & $75.5(7.1)(61-87)$ & $\mathrm{p}=0.730$ \\
Sex Male & $14(46.7 \%)$ & $8(32.0 \%)$ & $\mathrm{p}=0.269$ \\
$\quad$ Female & $16(53.3 \%)$ & $17(68.0 \%)$ & \\
Mean follow up (SD) (months) & $17.5(8.3)(6-36)$ & $20.9(8.7)(9-36)$ & $\mathrm{p}=0.145$ \\
Mean preoperative BCVA & $1.21(0.43)(0.30-1.80)$ & $1.28(0.42)(0.55-1.80)$ & $\mathrm{p}=0.569$ \\
$\begin{array}{l}(\mathrm{n}=54) \text { (logMAR (SD) } \\
\text { Mean postoperative BCVA }\end{array}$ & $0.50(0.36)(0.10-1.30)$ & $0.36(0.21)(0.10-1.00)$ & $\mathrm{p}=0.109$ \\
$(\mathrm{n}=54)$ (logMAR (SD) & & &
\end{tabular}

Group 1 = primary SFIOL implantation group; group 2 = secondary SFIOL implantation group; $\mathrm{BCVA}=$ best corrected visual acuity.

Values in parentheses were range.

cataratous lens because of unplanned ICCE. The mean time interval between cataract extraction and secondary SFIOL implantation was 9.6 (SD 11.7) months with a range of 1-38 months.

All SFIOLs implanted were Alcon CZ70BD SFIOLs (Alcon International, USA), which were single piece polymethylmethacrylate (PMMA) lenses with eyelets. The optic diameter was $7.0 \mathrm{~mm}$ and the overall diameter was $12.5 \mathrm{~mm}$. In all cases with vitreous loss, anterior vitrectomy would be performed. In primary SFIOL implantation the same corneal or scleral wound for cataract extraction was used, or the wound was enlarged to $8 \mathrm{~mm}$ in case of phacoemulsification. In secondary SFIOL implantation, a superior $8 \mathrm{~mm}$ corneal or scleral wound was made. The vast majority of surgeons used the ab externo technique modified from Lewis ${ }^{7}$ in the following manner. A double armed 10-0 polypropylene (Prolene) (W1713, Ethicon) suture with straight needles was used. Two fornix based conjunctival peritomy was made from 2-4 o'clock and 7-10 o'clock, respectively. The entry sites were marked $1.0-1.5 \mathrm{~mm}$ posterior to the limbus and $1.0 \mathrm{~mm}$ above or below the 3-9 o'clock horizontal meridian. The straight needle was passed through the sclera at the superior marking on one side and retrieved within the barrel of a 25 gauge needle inserted through the inferior marking on the opposite side. The same needle was inserted through the superior marking of the same side and retrieved through the inferior marking on the original side. The sutures were withdrawn through the corneal or scleral wound by a Sinky hook and cut accordingly. The two cut ends on each side passed through the corresponding eyelet and their ends tied. A square knot was made to join the two cut ends on each side and then rotated into the eye and out through the superior marking on each side. The IOL was inserted under viscoadaptive agent in the usual fashion, ${ }^{8}$ and the sutures were tightened and tied with open knots. The corneal or scleral wound was closed with interrupted 10-0 Nylon sutures (U7000 Ethilon, Ethicon). When IOP was adjusted to a normal level, the open knots were converted to closed knots and then rotated and buried through the inferior marking, which were the entry sites of the 25 gauge needle. The conjunctival peritomy was then closed.

Table 2 showed the early and late postoperative complications encountered after SFIOL surgery. For early complication within 1 month, there were 25 (83.3\%) eyes with a total of 55 complications in group 1; while there were 16 (64.0\%) eyes with a total of 26 complications in group 2. The average number of early complication per patient in group 1 was 1.70 (0.31) and that of group 2 was $1.09(0.22)$. Group 1 had a higher early complication rate and the difference between the groups was statistically significant (unpaired Student's $t$ test, $\mathrm{p}=0.028)$. Moreover, there are $22(73.0 \%)$ eyes complicated with corneal oedema in group 1 compared with 11 (44.0\%) eyes in group 2 and the difference was statistically significant $\left(\chi^{2}\right.$ test, $\left.p=0.027\right)$. For late complication after 1 month, there were $21(70.0 \%)$ eyes with a total of 37 complications in group 1, while there were $13(52.0 \%)$ eyes with a total of 19 complications in group 2 . The average number of late complication per patient in group 1 was $1.04(0.19)$ and that

Table 2 Postoperative complications encountered after scleral fixated intraocular lens (SFIOL) surgery $(n=55)$

\begin{tabular}{lccl}
\hline & $\begin{array}{l}\text { Primary SFIOL } \\
\text { (group 1, } \mathbf{n = 3 0 )}(\%)\end{array}$ & $\begin{array}{l}\text { Secondary SFIOL } \\
\text { (group 2, } \mathbf{n = 2 5} \text { ) (\%) }\end{array}$ & $\chi^{2}$ test \\
\hline Early complication (within 1 month) & & & \\
Fibrin & $6(20.0)$ & $1(4.0)$ & $\mathrm{p}=0.076$ \\
Increase IOP $>30 \mathrm{~mm} \mathrm{Hg}$ & $11(36.6)$ & $7(28.0)$ & $\mathrm{p}=0.495$ \\
Corneal oedema & $22(73.0)$ & $11(44.0)$ & $\mathrm{p}=0.027^{*}$ \\
Hyphaema & $8(26.6)$ & $4(16.0)$ & $\mathrm{p}=0.821$ \\
Vitreous haemorrhage & $8(26.6)$ & $3(12.0)$ & $\mathrm{p}=0.176$ \\
Total numbers of early complication & 55 & 26 & $\mathrm{p}=0.028^{*}$ \\
Late complication (after 1 month) & $5(16.7)$ & $3(12.0)$ & $\mathrm{p}=0.625$ \\
Glaucoma & $17(56.7)$ & $12(48.0)$ & $\mathrm{p}=0.521$ \\
Pupil deformation & $1(3.3)$ & 0 & $\mathrm{p}=0.357$ \\
Persistent uveitis (AC cells $\geqslant 1+$ ) & $2(6.7)$ & 0 & $\mathrm{p}=0.188$ \\
Cystoid macular oedema & $5(16.7)$ & $2(8.0)$ & $\mathrm{p}=0.337$ \\
Vitreous prolapse into anterior chamber & $1(3.3)$ & 0 & $\mathrm{p}=0.356$ \\
IOL decentration & $5(16.7)$ & $1(4.0)$ & $\mathrm{p}=0.134$ \\
IOL surface debris & $1(3.3)$ & $1(4.0)$ & $\mathrm{p}=0.896$ \\
Pseudophakic bullous keratopathy & 37 & 19 & $\mathrm{p}=0.077$ \\
Total numbers of late complication & & & \\
\hline *Statistically significant. & & &
\end{tabular}


of group 2 was $0.88(0.18)$. Group 1 also had a higher late complication rate but the difference between the groups was not statistically significant (unpaired Student's $t$ test, $p=0.077$ ). No cases of IOL removal, retinal break, or retinal detachment were encountered in this study.

One patient in group 1 had decreased vision because of anterior ischaemic optic neuropathy which is unrelated to surgery. In analysing the visual outcomes, this case was excluded. The mean preoperative logMAR BCVA in group 1 was 1.21 (SD 0.43) and that of group 2 was $1.28(0.42)$. The difference was not statistically significant (unpaired Student's $t$ test, $\mathrm{p}=0.569)$. The mean postoperative logMAR BCVA in group 1 was $0.50(0.36)$ and that in group 2 was $0.36(0.21)$. The difference was not statistically significant (unpaired Student's $t$ test, $\mathrm{p}=0.109$ ). In group $1,17(58.6 \%)$ eyes had 6/12 or better postoperative BCVA, nine $(31.0 \%)$ eyes had 6/15 to 6/60 BCVA, and three (10.3\%) eyes had less than 6/60 BCVA. In group 2, 19 (76.0\%) eyes had $6 / 12$ or better BCVA, and six $(24.0 \%)$ eyes had $6 / 15$ to $6 / 60$ BCVA. The difference in the number of eyes with BCVA of $6 / 12$ or better between the two groups was not statistically significant $\left(\chi^{2}\right.$ test, $\left.\mathrm{p}=0.177\right)$. In group $1,27(93.1 \%)$ eyes maintained or improved visual acuity, two (6.9\%) eyes lost BCVA (range -0.017 to -0.05 ). In group 2 all eyes maintained or improved BCVA. The difference was not statistically significant $\left(\chi^{2}\right.$ test, $\left.\mathrm{p}=0.181\right)$.

\section{DISCUSSION}

Malbran et al were the first to describe trans-sulcus scleral fixation of posterior chamber IOLs in aphakia eyes that had previously undergone ICCE in 1986. ${ }^{9}$ There were also a number of favourable reports on secondary SFIOL in the literature. ${ }^{10-15}$ However, to the best of our knowledge, no study has compared primary and secondary SFIOL implantations in relation to cataract surgery. In the absence of adequate lens capsular support in an eye during cataract extraction, a surgeon has three choices: primary implantation of ACIOL, primary implantation of SFIOL, and secondary implantation of ACIOL or SFIOL after a period of time. In a study by Bayramlar et al ${ }^{16}$ secondary implantation of flexible open loop ACIOL is shown to have more favourable visual outcomes and lower complication rates than primary ACIOL implantation. In this study, we compared the outcomes of primary and secondary SFIOL implantations.

Nineteen $(76.0 \%)$ eyes in our secondary implantation group (group 2) achieved postoperative BCVA of 6/12 or better. This result is comparable with previous studies that reported a rate of $57.1 \%$ to $92 \%$ in secondary SFIOL implantations..$^{1011} 1315$ The rate in the primary group (group 1 ) is not reported in the literature. In our study, such a rate was $58.6 \%$. It seems to be lower than that of group 2, although there was no statistically significant difference.

However, there were statistically significant higher complication rates in the early postoperative period in the primary SFIOL group. In particular, the rate of corneal oedema was higher in the primary SFIOL group and the difference was statistically significant. The rates of other complications were also higher in the primary SFIOL group but the differences were not statistically significant. Eyes undergoing primary SFIOL implantation may have a greater risk of postoperative inflammation with associated complications like cystoid macular oedema, as patients undergoing secondary SFIOL implantation are presumably a healthier group of eyes that have been preselected on the basis of their visual potential, and absence of inflammation, glaucoma, or anterior chamber abnormalities. Furthermore, the surgical technique of implantation of SFIOL requires elaborate skills and meticulous intraocular manipulation like anterior vitrectomy. This may not be ideally performed under the stressful situation associated with rupture of posterior capsule. This may partly explain the larger number of early complications in the primary implantation group.

There are more sight threatening complications such as corneal oedema, glaucoma, and cystoid macular oedema in group 1. This may be attributed to the prolonged surgical time in primary SFIOL implantation, as extra time is already used in the first part of the operation during cataract extraction. Prolonged operating time probably has a major role in light induced retinal injury, ${ }^{17}$ and such incidence from the operating microscope in cataract surgery ranged from $0 \%$ to $28 \%{ }^{18-20}$ Interestingly, the procedure of implantation of SFIOL may be especially vulnerable to light induced injury. In a study by Lanzetta et al, ${ }^{11}$ retinal photic injury visible with fluorescein angiogram were found in six (33\%) of 18 eyes undergoing secondary SFIOL. They proposed that during implantation of SFIOL, the eye lost the protective mechanisms against excessive light from the operating microscope reaching the posterior pole of the eye because of the dilated pupil and the loss of filtering effect of the crystalline lens during the time used in creating the scleral flap, suturing of the haptics of SFIOL, performing a meticulous vitrectomy, and implantation of the SFIOL.

Although primary implantation of SFIOL may have less favourable visual outcome and higher early complication rate, it has the benefit of one time operation and possibly shortening the total hospital stay. More importantly, a period of aphakia with poor vision after cataract extraction is avoided.

In conclusion, both primary and secondary implantations of SFIOL are suitable for treat aphakia and both methods are associated with favourable visual outcome. On the basis of this study secondary implantation of SFIOL seems to have a lower early complication rate than primary implantation. The retrospective nature of this study does not allow us to limit the number of surgeons. The experience of surgeons may affect the surgical outcome and the complication encountered. Further prospective studies including larger number of patients, more stringent criteria, and longer follow up are warranted.

\section{Authors' affiliations}

V Y W Lee, H K L Yuen, A K H Kwok, Department of Ophthalmology and Visual Sciences, The Chinese University of Hong Kong, Hong Kong Eye Hospital, Kowloon, Hong Kong, People's Republic of China A K H Kwok, Departments of Ophthalmology, Queen Mary Hospital and the Hong Kong Sanatorium and Hospital, .Hong Kong, People's Republic of China

Financial and proprietary interest: Nil.

Financial support: Nil.

Correspondence to: Dr Alvin K H Kwok, Department of Ophthalmology, Hong Kong Sanatorium and Hospital, 2 Village Road, Happy Valley, Hong Kong; alvinkwok@hksh.com

Accepted for publication 17 March 2003

\section{REFERENCES}

1 Bellucd R, Pucci V, Morselli S, et al. Secondary implantation of anglesupported anterior chamber and scleral-fixated posterior chamber intraocular lenses. J Cataract Refract Surg 1996;22:247-52.

2 Miyake K, Asakura M, Kobayashi H. Effect of intraocular lens fixation on the blood-aqueous barrier. Am J Ophthalmol 1984;98:451-5.

3 Apple D, Mamalis N, Lofffield K, et al. Complications of intraocular lenses: a historical and histopathological review. Surv Ophthalmol 1984;29:|-54.

4 Stark WJ, Gottsch JD, Goodman DP, et al. Posterior chamber intraocular lens implantation .in the absence of capsular support. Arch Ophthalmol 1989;107:1078-83.

5 Holladay JT. Evaluating the intraocular lens optic. Surv Ophthalmol 1986:30:385-90. 
6 Appel SD, Brilliant RL. The low vision examination. In: Brilliant RL, ed. Essentials of low vision practice. Chapter 3. Boston: Butterworth-Heinemann, 1999:20-46

7 Lewis JS. Ab externo sulcus fixation. Ophthalmic Surg 1991;22:692-5.

8 Lewis JS. Sulcus fixation without flaps. Ophthalmology 1993;100:1346-50.

9 Malbran ES, Malbran E Jr, Negri I. Lens guide suture for transport and fixation in secondary $\mathrm{IOL}$ implantation after intracapsular extraction. Int Ophthalmol 1986;9:151-60

10 Lanzetta $P$, Bandello FM, Virgili $G$, et al. Is scleral fixation a safe procedure for intraocular lens implantation? Doc Ophthalmol 1999;97:317-324.

11 Lanzetta P, Menchini U, Virgili G, et al. Scleral fixated intraocular lenses: an angiographic study. Retina 1998;18:515-20.

12 Uthoff D, Teichmann KD. Secondary implantation of scleral-fixated intraocular lenses. J Cataract Refract Surg 1998;24:945-50.

13 Kershner RM. Simple method of transscleral fixation of a posterior chamber intraocular lens in the absence of the lens capsule. J Refract Corneal Surg 1994; 10:647-51
14 McCluskey P, Harrisberg B. Long-term results using scleral-fixated posterior chamber intraocular lenses. J Cataract Refract Surg 1994;20:34-9.

15 Hahn TW, Kim MS, Kim JH. Secondary intraocular lens implantation in aphakia. J Cataract Refract Surg 1992;18:174-179.

16 Bayramlar HS, Hepsen IF, Cekic O, et al. Comparison of the results of primary and secondary implantation of flexible open-loop anterior chamber intraocular lens. Eye 1998;12:826-8.

17 Azzolini C, Brancato R, Venturi G, et al. Updating on intraoperative light induced retinal injury. Int Ophthalmol 1994;18:269-76.

18 Byrnes GA, Chang B, Loose I, et al. Prospectic incidence of photic maculopathy after cataract surgery. Am J Ophthalmol 1995;1 19:231-322.

19 Khwarg SG, Linstone FA, Daniels SA, et al. Incidence, risk factors, and morphology in operating microscope light retinopathy. Am J Ophthalmol 1987; 103:255-63.

20 Byrnes GA, Antoszyk AN, Mazur DO, et al. Photic maculopathy after extracapsular cataract surgery: a prospective study. Ophthalmology 1992;99:731-7. 